\title{
Excited-state intramolecular proton transfer in jet-cooled 3-hydroxyflavone. Deuteration studies, vibronic double-resonance experiments, and semiempirical (AM1) calculations of potential-energy surfaces
}

\author{
A. Mühlpfordt a , T. Bultmann ${ }^{\text {b }}$, N.P. Ernsting ${ }^{\text {b,* }}$ \\ - Abteilung Spektroskopie, Max-Planck-Institut für biophysikalische Chemie, Postfach 2841, 37018 Göttingen, Germany \\ b Abteilung Laserphysik, Postfach 2841, 37018 Göttingen, Germany
}

and

B. Dick

Institut für physikalische und theoretische Chemie, Universität Regensburg, Postfach 397, 93053 Regensburg, Germany

Received 5 October 1993

\begin{abstract}
3-Hydroxyflavone (3-HF) and 3-deuteroxyflavone (3-DF) were examined by fluorescence excitation spectroscopy in a supersonic free jet. Compared to 3-HF, vibronic bands of 3-DF are significantly narrower. Substitution of H by D also appears to split vibronic bands into at least three bands leading to a congested spectrum. Fluorescence-dip double-resonance spectroscopy revealed that the complicated spectrum of 3-DF consists of at least three independent partial spectra which are superimposed. The vibrational pattern of every partial spectrum is identical to that of 3-HF, but partial spectra differ in their spectral position. Semiempirical calculations (MNDO/AM1 with limited CI) were used to generate ground- and singlet excited-state potential energy surfaces as a function of phenyl and hydroxyl torsional angles. The deuteration effects suggest the existence of potential energy barriers to low-frequency hydroxyl hydrogen motion, with barrier height on the order of the vibrational zero-point energy.
\end{abstract}

\section{Introduction}

The yellow flower petals of many plants owe the brilliance of their colors to derivatives of the parent compound 3-hydroxyflavone (3-HF, cf. fig. 1). In aliphatic solutions, the absorption spectrum of 3-HF has its first peak in the near-UV at $352 \mathrm{~nm}$ while the fluorescence is centered around $520 \mathrm{~nm}$ in the yellow-green spectral region, i.e. the Stokes shift exceeds $8000 \mathrm{~cm}^{-1}$.

This anomalous fluorescence behavior has been studied over several decades and may be explained by an intramolecular proton transfer reaction, just as in the historic case of salicylic acid [2]. The corresponding Förster reaction cycle for 3-HF is shown in fig. 1. In nonpolar solvents, the most stable form of

\footnotetext{
* Corresponding author.
}

the molecule in its electronic ground state $S_{0}$ contains an intramolecular hydrogen bond between the 3-hydroxy group and the carbonyl group. After absorption of a UV photon, the normal form in its first excited singlet state $S_{1}$ is not longer stable. Instead, a charge redistribution causes an intramolecular proton transfer, which leads to a tautomeric form $S_{1}^{\prime}$ of the molecule in its electronically excited singlet state. The tautomeric form of 3-HF has zwitterionic character and is in turn unstable in the electronic ground state $S_{0}^{\prime}$. Therefore, fluorescence emission in the yellow-green spectral region is followed by reverse proton transfer, and the reaction cycle is thus closed.

A key element in the overall photochemical reaction cycle is excited-state intramolecular proton transfer (ESIPT) in the singlet manifold, i.e. the radiationless process $S_{1} \rightarrow S_{1}^{\prime}$. This elementary reaction has been studied for many molecules and the results 


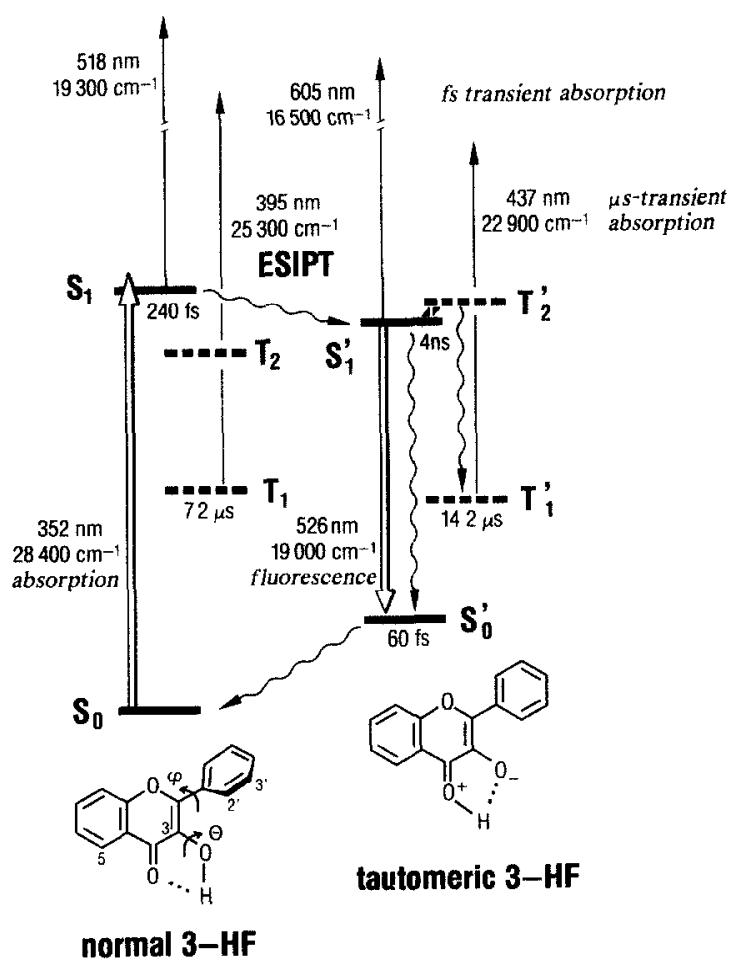

Fig. 1. Electronic states, optical transitions, and radiationless processes which are relevant for 3-hydroxyflavone.

have been reviewed comprehensively [3]. In particular, interest in 3-HF was revived when Sengupta and Kasha observed dual fluorescence at low temperature in aliphatic glasses: UV fluorescence which was presumed to have originated from the normal excited tautomer, and green fluorescence from the proton-transferred tautomer [4]. In explanation, the authors formed the intriguing hypothesis that proton transfer would be possible only when the phenyl group is planar with the 3-hydroxychromone moiety $(=3$ HF with the phenyl ring removed; see fig. 1). A rigid matrix would hinder some molecules from attaining torsional planarity; the ESIPT reaction would thus be impeded and normal fluorescence should be observed.

Of the intense research into the spectroscopy and photochemistry of 3-HF and closely related compounds that followed this hypothesis, we will sketch the major lines of work, briefly discussing a few contributions only. We refer the reader to the full literature cited there.

\subsection{Ground state $\left(S_{0}\right)$ molecular structure and solvent complexes}

In the gas phase at temperatures between $320-400$ $\mathrm{K}$, only tautomer fluorescence was observed [5]. Pressure studies on hydrocarbon solutions at room temperature showed that, in contradiction to the original hypothesis, viscosity had no effect on the ESIPT reaction [6]. Using extremely dry methyl-cyclohexane glasses at $77 \mathrm{~K}$ [7] or Shpol'skii matrices [8] it was eventually realized that complexes of 3HF with hydrogen-bonding solvent impurities had been responsible for the observed normal fluorescence. Now an extremely low quantum yield of normal fluorescence from the intramolecularly $\mathrm{H}$-bonded form could be demonstrated even at low temperaturcs indicating fast competing ESIPT. These findings were corroborated for $3-\mathrm{HF}$ which had been isolated in an argon matrix at $10 \mathrm{~K}[9,10]$. The crystal structures of 3-HF and its 2'- and 3'-methylated derivatives all possess the cyclic intramolecular hydrogen bond between the carbonyl group and the 3-hydroxy group [11]. Specific complexes of 3-HF with small protic solvent molccules (water, methanol, ammonia) have been prepared in argon matrices. They were characterized by fluorescence and its excitation spectroscopy as well as by picosecond kinetics of ESIPT [10,12]. Recently, isolated and well-defined complexes $3-\mathrm{HF} \ldots\left(\mathrm{H}_{2} \mathrm{O}\right)_{n}$ with $n=1,2$ have been observed in a supersonic jet [13].

\subsection{Vibrational and vibronic structure of $3-H F$ in its normal form}

The infrared absorption spectrum of 3-HF in an argon matrix showed a doublet structure for bands which could be assigned to an $\mathrm{OH}$ stretching mode (at $3322.7 / 3333.3 \mathrm{~cm}^{-1}$ ) or to an in-plane $\mathrm{OH}$ bending mode (at $1340.5 / 1338.5 \mathrm{~cm}^{-1}$ ) [14]. Vibrational information about the original, normal form of the molecule in its primary excited state $S_{1}$ was obtained from fluorescence excitation spectroscopy of isolated, cold $3-\mathrm{HF}$ in a supersonic jet [15]. No significant "normal" UV fluorescence could be observed in these experiments, which is in agreement with the matrix results. The excitation spectrum was shown to be characterized by a progression in a mode of $45 \mathrm{~cm}^{-1}$. This mode was not observed in the cor- 
responding 3-hydroxychromone which lacks the phenyl group [13]. Returning to $3-\mathrm{HF}$, the $45 \mathrm{~cm}^{-1}$ mode must then be assigned to phenyl torsion. Its vibronic bands were best characterized by Lorentzian lineshapes with homogeneous widths (fwhm) in the range $4.1-2.6 \mathrm{~cm}^{-1}$ corresponding to vibronic lifetimes in the range 1.3-2.0 ps. On the basis of molecular structures from semiempirical MNDO/AM1 calculations, it has been suggested that a bending mode modulates the intramolecular proton transfer distance and thus acts as a promoting mode for the non-radiative process [16].

\subsection{Excited-state intramolecular proton transfer $S_{1} \rightarrow S_{1}^{\prime}$}

Many attempts at a direct, time-resolved observation of the process $S_{1} \rightarrow S_{1}^{\prime}$ were aimed at 3-HF which has a preformed intramolecular $\mathrm{H}$-bond ${ }^{\# 1}$, typically by following the rise of tautomeric fluorescence with a streak camera. The early (pre-84) experiments gave results which were complicated by the extreme sensitivity to solvent impurities mentioned above. Following the discovery of this solvent effect, the tautomer fluorescence rise could then be used to study structural rearrangements in protic [17] and aprotic polar solvents [18-20]. However in this paper we are mainly interested in weakly solvated or isolated 3-HF and its intrinsic photochemical reactions. Therefore let us return to 3-HF embedded in an argon matrix at $10 \mathrm{~K}$, where the largest observed lower limit to the intrinsic ESIPT rate constant is $k_{\text {ESIPT }}>5 \times 10^{11} \mathrm{~s}^{-1}$ [9]. Under the same conditions a kinetic study of spectral components of the tautomer fluorescence band revealed a spectral relaxation with a time constant of approximately $60 \mathrm{ps}$ [20]. This observation was interpreted as torsional relaxation following fast proton transfer.

A further characterization of the educt and product states, $S_{1}$ and $S_{1}^{\prime}$, may be obtained from transient optical absorption spectroscopy in protic solvents with picosecond time resolution [21]. Two absorption bands at $518 \mathrm{~nm}$ and at $605 \mathrm{~nm}$ were assigned to excited-state absorption from $S_{1}$ and from $S_{1}^{\prime}$, respec-

\footnotetext{
\#1 We will use the term intrinsic reaction for the elementary step leading from an educt which exists with an internal $\mathrm{H}$-bond to the corresponding product.
}

tively, while a gain band at $540 \mathrm{~nm}$ corresponds to stimulated emission $S_{1}^{\prime} \rightarrow S_{0}^{\prime}$ (cf. fig. 1). In the experiment with the best time resolution to date, the excited-state absorption at $620 \mathrm{~nm}$ due to the protontransferred tautomeric product state $S_{1}^{\prime}$ has been used to monitor the ESIPT dynamics at room temperature in methyl cyclohexane [22]. In this case the intrinsic reaction was shown to have a time constant of $240 \mathrm{fs}$, in the order of the period for low-frequency skeletal vibrations. Proton transfer was depicted as part of the evolution of a vibrational wavepacket on a multidimensional $S_{1}$ potential energy surface without barrier on the way to the tautomeric form.

\subsection{Properties of the tautomeric excited state $S_{I}^{\prime}$}

The lifetime of the tautomeric state has been obtained in various solvents at room temperature as summarized in ref. [23]. To our knowledge, only few systematic studies of rate constants for the nonradiative deactivation $S_{1}^{\prime} \rightarrow S_{0}^{\prime}$ in nonpolar solvents have been made $[24,25]$. The following activation energies have been reported: $1430 \mathrm{~cm}^{-1}$ in 2-methyltetrahydrofuran, $2480 \mathrm{~cm}^{-1}$ in methylcyclohexane, and $3010 \mathrm{~cm}^{-1}$ in 3-methylpentane. In addition, the nonradiative decay rate was shown to decrease with increasing hydrostatic pressure in nonpolar solvents [26].

\subsection{Triplet states $T_{1}$ and $T_{1}^{\prime}$}

Transient absorption studies on a microsecond time scale showed two absorption bands, at $395 \mathrm{~nm}$ with a $7.2 \mu$ s decay time and at $437 \mathrm{~nm}$ with a decay time constant of $14.2 \mu \mathrm{s}$ [27-29]. Both transients were quenched by oxygen, with slightly different secondorder rate constants. Optical excitation of the second band (two-step laser excitation, TSLE) produced tautomer fluorescence. On the basis of semiempirical calculations for both tautomeric forms with either singlet or triplet multiplicity, these intriguing observations could be rationalized in terms of triplet-triplet absorption as also indicated in fig. 1 [23]. Stimulated-emission pumping of the transition $S_{1}^{\prime} \rightarrow S_{0}^{\prime}$ reduced the fluorescence from TSLE at $437 \mathrm{~nm}$ [30], since the state $T_{1}^{\prime}$ which is responsible for the $437 \mathrm{~nm}$ absorption band was bypassed in this way. 


\subsection{Ground-state reverse intramolecular proton transfer $S_{0} \leftarrow S_{0}^{\prime}$}

The tautomeric ground state $S_{0}^{\prime}$ is accessible by radiative transition from the excited singlet state $S_{1}^{\prime}$. Hence the dispersion of the tautomeric fluorescence into well-resolved vibronic lines should, in principle, yield the pertinent $S_{0}^{\prime}$ vibrational structure while the linewidth should give the vibrational lifetime in the absence of pure dephasing, for instance at very low temperatures in inert media. The best fluorescence spectrum of 3-HF in solid argon (at $30 \mathrm{~K}$ ) [10] shows broad spectral structure. Deuteration resulted in sharper spectral features of the corresponding 3-DF with indications of inhomogeneous broadening (site distribution ). From the linewidths, a lower limit of the vibronic lifetimes of 60 and $260 \mathrm{fs}$ was estimated for 3-HF and 3-DF, respectively. This short vibronic lifetime indicates an ultrafast reverse proton transfer in the electronic ground state. Consequently it was not possible to accumulate the ground-state tautomer under these conditions [14].

A fast population of $S_{0}^{\prime}$ was also achieved by stimulated-emission pumping [31]. In this experiment, which had a time resolution of $30 \mathrm{ps}$, the reverse proton transfer could not be resolved.

In section 3 of the present paper, we report experiments with 3-HF and 3-DF cooled and isolated in a supersonic jet. Our first aim was simply to obtain the fluorescence excitation spectrum of deuterated 3-DF and to compare line positions and linewidths to those of the protonated compound. It has already been mentioned by Itoh et al. [13] that H/D substitution leads to a spectrum which is not merely shifted but also more complex that that of 3-HF. We have therefore broken this complicated spectrum into a sum of independent partial spectra by using the fluorescence-dip double-resonance technique.

In the theoretical section 4 , we investigate the ground-state potential energy surface by semiempirical MNDO/AM1 calculations. The major conclusion is that - in addition to the long list of topics which has been reviewed for most of this introduction subtle details of the potential energy surface and the possibility of ground-state isomers should be considered in explaining high-resolution excitation spectra.

\section{Experimental methods}

\subsection{Sample preparation}

3-HF was supplied from Aldrich. For the spectroscopy of 3-HF, the compound was purified by sublimation followed by zone melting; the final melting point was $170.6^{\circ} \mathrm{C}$.

For deuteration, $3.4 \mathrm{~g}\left(14.3 \times 10^{-3} \mathrm{~mol}\right)$ of $3-\mathrm{HF}$ (as supplied) were extracted from a glass frit by refluxing with $50 \mathrm{~g}(1.5 \mathrm{~mol})$ of deuterated methanol ( $98 \%$ MeOD, from Aldrich). The extraction typically required three days and was carried out under a constant stream of dry nitrogen. The $\mathrm{MeOH} / \mathrm{MeOD}$ mixture was then distilled off and replaced by fresh MeOD. After refluxing again for three days, the crystals were filtered and dried on a vacuum line. All handling of the compound was carried out in a wellmaintained drybox flushed with argon. Finally, the material was sublimated under high vacuum $\left(\approx 10^{-3}\right.$ mbar, $140^{\circ} \mathrm{C}$ ). The white 3-DF thus obtained was stored in the drybox. Purity was checked by MS and NMR, and the degree of deuteration was determined to be better than $90 \%$. Before each jet experiment, some $20 \mathrm{mg}$ of 3-DF were placed into a glass transfervessel which was then sealed and connected to the jet nozzle system. After evacuation of the vessel, the crystals could be dropped onto a glass frit which was placed in the stagnation chamber just before the nozzle.

\subsection{Experimental setup}

The experimental setup is partly shown in fig. 2 . The supersonic jet was formed by an expansion of neon, at stagnation pressure of 1.0 bar and temperature of $140^{\circ} \mathrm{C}$, through a glass nozzle with $120 \mu \mathrm{m}$ diameter. Fluorescence was excited $2.8 \mathrm{~mm}$ downstream by a tunable pulsed dye laser (Lambda Physik FL2001) which is labelled as "probe" dye laser in the figure. The emission was collected at right angles to the jet and laser beam by an aspheric quartz lens in combination with a spherical mirror. The tautomer fluorescence was selected by a broad-band interference filter centered at $500 \mathrm{~nm}$ with a full bandpass of $100 \mathrm{~nm}$. No signal above noise was observed when the region $380-420 \mathrm{~nm}$ was selected, either for 3-DF or for 3-HF. Therefore the use of an interference fil- 


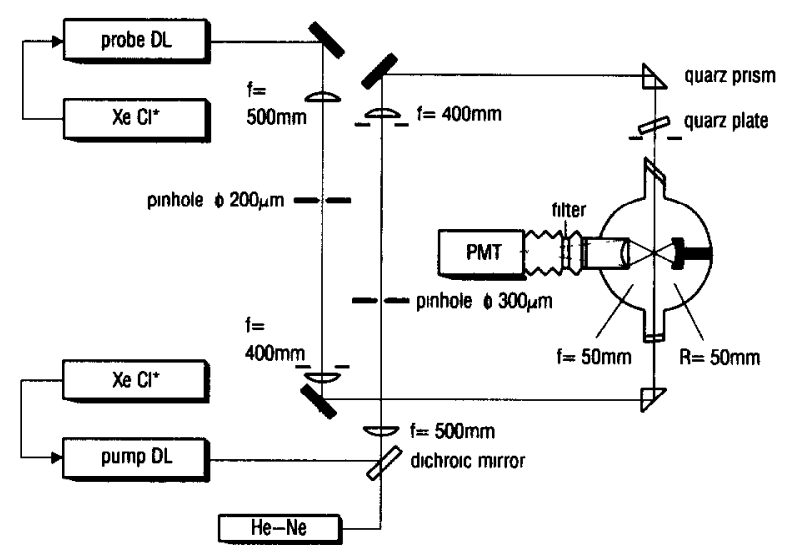

Fig. 2. Experimental setup for laser-induced fluorescence and fluorescence-dip double-resonance spectroscopy in a supersonic free jet.

ter centered at $500 \mathrm{~nm}$ was not essential in our experiments and simply served to reject stray light. Excitation wavenumbers refer to measurement in vacuo. The error in absolute and relative line position is estimated to be better than $\pm 0.5 \mathrm{~cm}^{-1}$.

A second dye laser (Lambda Physik LPD3000) was required for labelling selected vibronic transitions. This second laser is termed "pump" dye laser in fig. 2. It was aligned so that its focal region fully overlapped the focal region of the probe laser. In order to facilitate this alignment in a well-controlled manner, we chose an imaging geometry which is also shown in fig. 2. Each dye laser beam was focused softly (with a lens of long focal length) onto a pinhole, and these pinholes were then imaged to the region just below the jet nozzle. By choosing appropriate pinhole diameters and lens positions, the diameters of the pumped and probed volumes were set to 840 and 220 $\mu \mathrm{m}$, respectively. A thick and slightly wedged quartz plate in the pump beam could be tilted in order to translate the pump beam parallel to the probe beam for an optimal signal (see below).

For a particular labelling experiment, the probe laser was fixed at the wavelength for the peak of the chosen vibronic band and its laser-induced fluorescence was monitored selectively through an appropriate time gate. The pump laser was fired several hundred ns prior to the probe laser, and it was tuned across the spectral region of interest. When the pump comes into resonance with a transition which shares a common ground state with the probed transition, ground state population is removed, and this then registers as a dip in the monitored fluorescence. The pump and probe pulse energies in our experiments were typically 400 and $100 \mu \mathrm{J}$, respectively.

\section{Experimental results}

\subsection{Laser-induced fluorescence of jet-cooled 3-DF}

Fig. 3 compares the fluorescence excitation spectra of jet-cooled 3-HF (top) and 3-DF (bottom). Only the incipient part of the line spectra is shown in each case, up to an excess vibrational energy of $\approx 150$ $\mathrm{cm}^{-1}$. Not shown in the figure is an unstructured background which underlies each spectrum and extends far to the red of the first observed line. An unstructured background to the fluorescence excitation

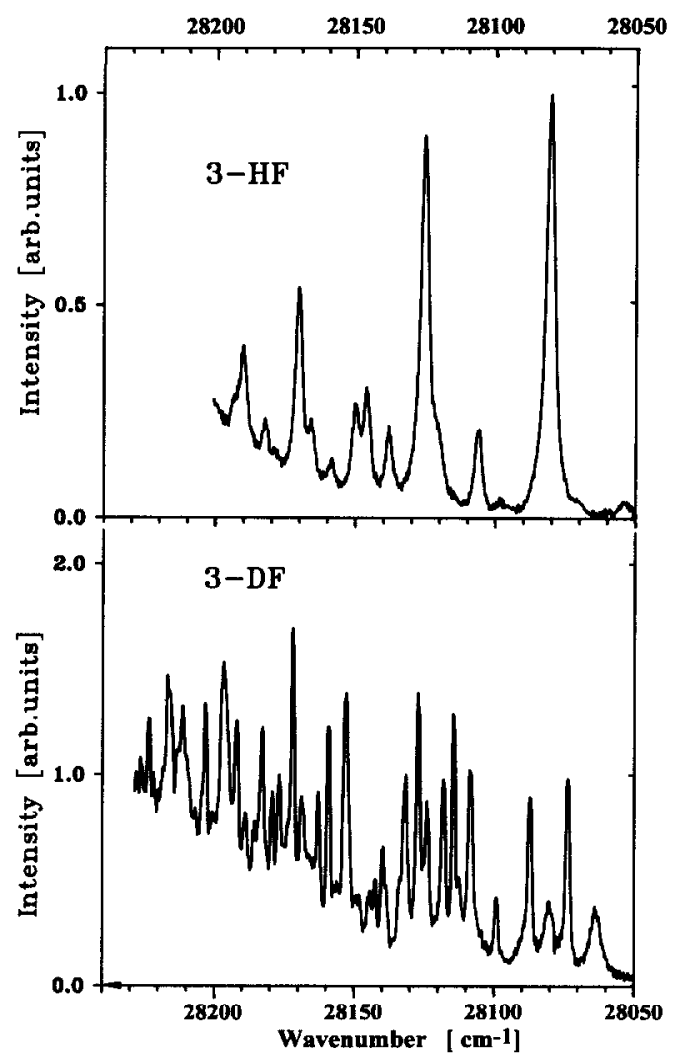

Fig. 3. Fluorescence excitation spectrum of jet-cooled 3-HF and 3-DF. 
spectra of jet-cooled 3-HF has been reported before [15]; it depends sensitively on stagnation conditions and water content [13] of the carrier gas. In order to represent the vibronic structure only, the following procedure was applied to the recorded fluorescence excitation spectra: (i) an appropriate constant value representing the background was subtracted from the recorded data, and (ii) the result was normalized to the peak intensity of the first prominent line.

$H / D$ substitution in 3-hydroxyflavone leads to a complicated vibronic spectrum which is shown in the lower half of the figure. Deuteration also results in a reduction of vibronic linewidth. This is demonstrated in fig. 4 , which shows an enlargement of the region of the spectral origin (dots representing measurement points). The 00 band of $3-\mathrm{HF}$ at 28080.3 $\mathrm{cm}^{-1}$ has a Lorentzian lineshape (full line); its fwhm

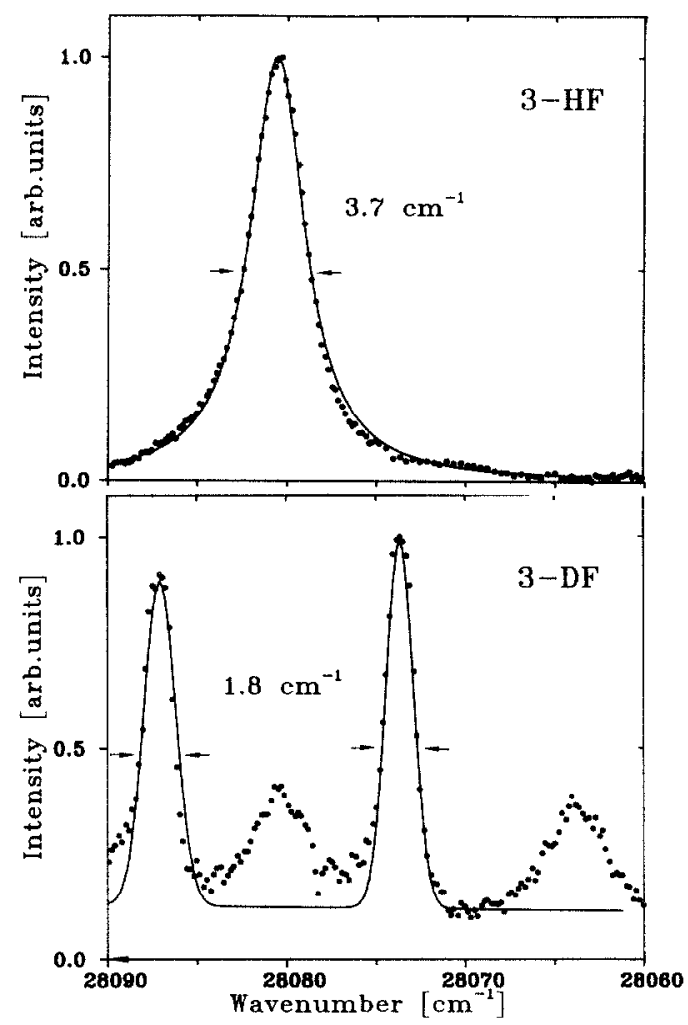

Fig. 4. Comparison of vibronic bands for 3-HF (top) and 3-DF (bottom). Dots refer to measurement points and solid lines to fits by a Lorentzian (top) or Gaussian (bottom) lineshape function. In the case of 3-DF, only the narrow bands have been simulated. was determined to be $3.7 \mathrm{~cm}^{-1}$ in this experiment. Values of $4.1 \mathrm{~cm}^{-1}$ [15] and $3.5 \mathrm{~cm}^{-1}$ [13] have been reported previously. This band is also observed in the 3-DF spectrum but with reduced relative amplitude, and it is due to undeuterated 3-HF in the sample $(\approx 10 \%$, see below $)$. The broad band at $28064.1 \mathrm{~cm}^{-1}$ in the lower spectrum has no correspondence in the 3-HF spectrum and it must therefore also be assigned to 3-DF. The narrow bands in the 3-DF spectrum were fitted with a Gaussian lineshape function (full line) with fwhm of $1.8 \mathrm{~cm}^{-1}$ representing the convolution of rotational envelope and laser excitation lineshape. Their line positions and relative peak intensities are collected in table 1. Back-exchange 3-DF $\rightarrow$ 3-HF occurs rapidly if the sample in the stagnation chamber is exposed to (moist) air during a jet experiment. Depending on exposure time (several seconds up to a minute), the spectrum of 3-HF is partially or fully recovered. Thus partial back-exchange may easily cause experimental artifacts. Brief exposure to moisture leads initially to a surface layer of 3-HF on bulk crystals of 3-DF. As the evaporation of the solid sample continues during the experiment, the 3-HF/3-DF ratio changes, resulting in spectra which then depend on scan time.

\subsection{Fluorescence-dip double-resonance spectroscopy of 3-DF}

The fluorescence-excitation spectrum of 3-DF from fig. 3 is reproduced in the bottom half of fig. 5. Double-resonance experiments are needed to unravel the complexity of this spectrum. For example, the top half of fig. 5 shows a typical fluorescence-dip spectrum of 3-DF. In this case the probe laser was set to the sharp band at $28087.1 \mathrm{~cm}^{-1}$ (band $b$ in fig. 5B), the corresponding fluorescence emission was monitored, and the pump laser was scanned. A fluorescence dip in fig. 5A either corresponds to a single band in the total spectrum of fig. $5 \mathrm{~B}$, or else it marks a component of a superposition of vibronic bands. In this way, a vibronic pattern is obtained which has been indicated along a dashed bar in fig. 5B. Bands belonging to such a pattern are related through a common vibrational ground state for the corresponding optical transitions. Monitoring other bands ( $a$ or $\mathrm{c}$ ), we obtained two other patterns which are indicated along two solid bars, respectively. Of the bands in the total spectrum 
Table 1

Vibronic bands in the fluorescence-excitation spectrum of 3-DF and their assignment to different partial spectra

\begin{tabular}{|c|c|c|c|c|c|c|c|c|c|}
\hline \multirow[t]{2}{*}{$\begin{array}{l}\text { Wavenumber } \\
\left(\mathrm{cm}^{-1}\right)\end{array}$} & \multicolumn{3}{|l|}{$\begin{array}{l}\text { Probe } \nu(\mathrm{a})_{0} \\
28073.7 \mathrm{~cm}^{-1}\end{array}$} & \multicolumn{3}{|l|}{$\begin{array}{l}\text { Probe } \nu(\mathrm{b})_{0} \\
28087.1 \mathrm{~cm}^{-1}\end{array}$} & \multicolumn{3}{|l|}{$\begin{array}{l}\text { Probe } \nu(\mathrm{c})_{0} \\
28108.3 \mathrm{~cm}^{-1}\end{array}$} \\
\hline & $\begin{array}{l}\text { wavenumber } \\
\left(\mathrm{cm}^{-1}\right)\end{array}$ & $\begin{array}{l}\Delta \nu \\
\left(\mathrm{cm}^{-1}\right)\end{array}$ & assignment & $\begin{array}{l}\text { wavenumber } \\
\left(\mathrm{cm}^{-1}\right)\end{array}$ & $\begin{array}{l}\Delta \nu \\
\left(\mathrm{cm}^{-1}\right)\end{array}$ & assignment & $\begin{array}{l}\text { wavenumber } \\
\left(\mathrm{cm}^{-1}\right)\end{array}$ & $\begin{array}{l}\Delta \nu \\
\left(\mathrm{cm}^{-1}\right)\end{array}$ & assignment \\
\hline \multicolumn{10}{|l|}{28064.1} \\
\hline \multicolumn{10}{|l|}{28080.3} \\
\hline 28087.1 & & & & 28087.1 & 0.0 & $\nu(\mathrm{b})_{0}$ & & & \\
\hline 28099.1 & 28099.8 & 26.1 & $\nu(\mathrm{a})_{1}$ & & & & & & \\
\hline 28108.3 & & & & & & & 28108.3 & 0.0 & $\nu(\mathrm{c})_{0}$ \\
\hline 28112.5 & 28113.2 & 39.5 & $\nu(\mathrm{a})_{2}$ & 28112.7 & 25.6 & $\nu(b)_{1}$ & & & \\
\hline \multicolumn{10}{|l|}{28114.8} \\
\hline 28118.2 & 28118.0 & 44.3 & $\nu(a)_{3}$ & & & & & & \\
\hline \multicolumn{10}{|l|}{28124.1} \\
\hline 28127.3 & & & & 28127 & 40.7 & $\nu(\mathrm{b})_{2}$ & & & \\
\hline 28131.7 & 28131.0 & 57.3 & $\nu(\mathrm{a})_{4}$ & 28132.5 & 45.4 & $\nu(\mathrm{b})_{3}$ & 28134.8 & 26.5 & $\nu(c)_{1}$ \\
\hline \multicolumn{10}{|l|}{28133.6} \\
\hline 28139.7 & 28138.5 & 64.8 & $\begin{array}{l}\nu(\mathrm{a})_{1}+ \\
\nu(\mathrm{a})_{2}\end{array}$ & & & & & & \\
\hline 28142.4 & 28142.9 & 69.2 & $\begin{array}{l}\nu(\mathrm{a})_{1}+ \\
\nu(\mathrm{a})_{3}\end{array}$ & & & & & & \\
\hline 28144.4 & & & & 28144.4 & 57.3 & $\nu(\mathrm{b})_{4}$ & 28148.5 & 40.2 & $\nu(\mathrm{c})_{2}$ \\
\hline 28149.2 & 28151.2 & 77.5 & $\nu(\mathrm{a})_{5}$ & & & & 28153.8 & 45.5 & $\nu(\mathrm{c})_{3}$ \\
\hline 28152.8 & & & & 28152.3 & 65.2 & $\begin{array}{l}\nu(\mathrm{b})_{1}+ \\
\nu(\mathrm{b})_{2}\end{array}$ & & & \\
\hline 28158.9 & 28158.4 & 84.7 & $\begin{array}{l}\nu(\mathrm{a})_{2}+ \\
\nu(\mathrm{a})_{3}\end{array}$ & 28157.1 & 70.0 & $\begin{array}{l}\nu(\mathrm{b})_{1}+ \\
\nu(\mathrm{b})_{3}\end{array}$ & & & \\
\hline 28162.9 & 28163.9 & 90.2 & $2 \nu(\mathrm{a})_{3}$ & 28165.8 & 78.7 & $\nu(\mathrm{b})_{5}$ & 28166.5 & 58.2 & $\nu(\mathrm{c})_{4}$ \\
\hline \multicolumn{10}{|l|}{28168.8} \\
\hline 28172.0 & & & & 28172.2 & 85.1 & $\begin{array}{l}\nu(\mathrm{b})_{2}+ \\
\nu(\mathrm{b})_{3}\end{array}$ & & & \\
\hline 28173.6 & & & & & & & 28173.6 & 65.3 & $\begin{array}{l}\nu(\mathrm{c})_{1}+ \\
\nu(\mathrm{c})_{2}\end{array}$ \\
\hline \multicolumn{9}{|l|}{28179.3} & \\
\hline 28182.8 & 28183.0 & 109.3 & $\begin{array}{l}\nu(\mathrm{a})_{1}+ \\
\nu(\mathrm{a})_{2}+ \\
\nu(\mathrm{a})_{3}\end{array}$ & & & & & & \\
\hline 28185.6 & 28186.8 & 113.1 & $2 \nu(a)_{4}$ & & & & 28187.6 & 79.3 & $\nu(\mathrm{c})_{5}$ \\
\hline \multirow{2}{*}{$\begin{array}{l}28189.0 \\
28191.9\end{array}$} & & & & 28188.8 & 101.7 & $\nu(\mathrm{b})_{6}$ & & & \\
\hline & & & & & & & 28194.3 & 86.0 & $\begin{array}{l}\nu(\mathrm{c})_{2}+ \\
\nu(\mathrm{c})_{3}\end{array}$ \\
\hline 28196.7 & & & & 28196.8 & 109.7 & $\begin{array}{l}\nu(\mathrm{b})_{1}+ \\
\nu(\mathrm{b})_{2}+ \\
\nu(\mathrm{b})_{3}\end{array}$ & & & \\
\hline 28199.9 & & & & 28200.0 & 112.9 & $2 \nu(b)_{4}$ & 28198.7 & 90.4 & $2 \nu(\mathrm{c})_{3}$ \\
\hline
\end{tabular}




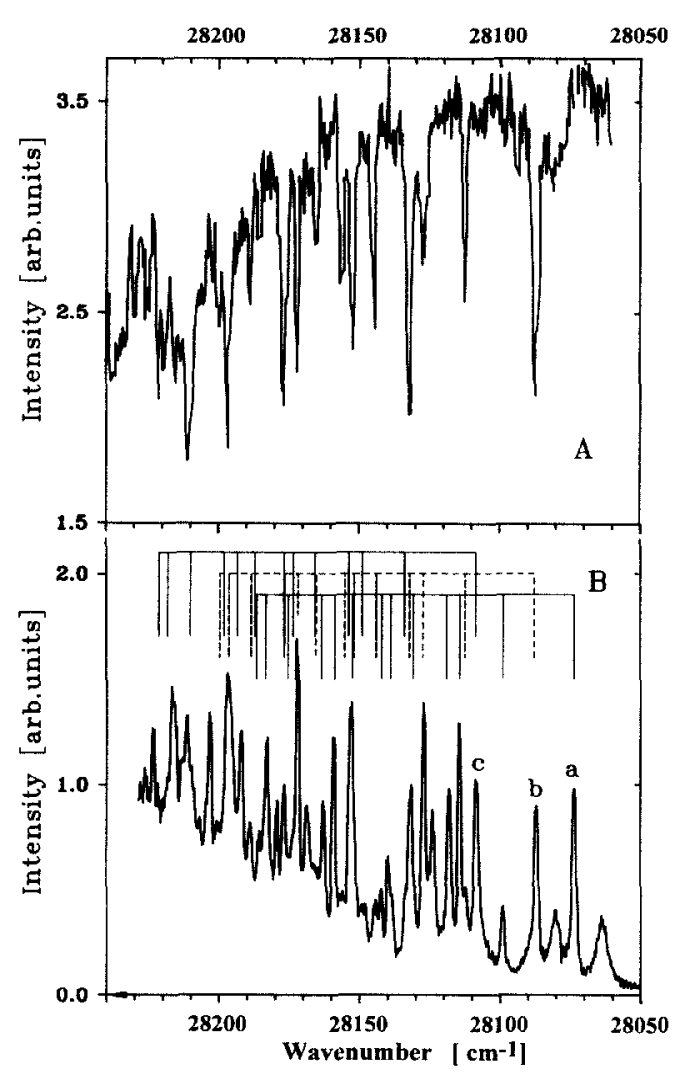

Fig. 5. (A) Fluorescence-dip spectrum of 3-DF monitoring band b. (B) Assignment of bands in the excitation spectrum of 3-DF based on fluorescence-dip spectroscopy.

of $3-D F, 80 \%$ are accounted for in this way. These assignments are also collected in table 1 . One other relatively broad band, at $28064.1 \mathrm{~cm}^{-1}$, is also assigned to 3-DF. A pattern built upon this broad band is probably obscured by the congested structure due to the other vibronic patterns.

Next we compare the three vibronic patterns shown in fig. 5B. Their spectral origins are different but the spectral separations are identical. Also, the spectral separations match precisely the spectral separations in the fluorescence-excitation spectrum of undeuterated 3-HF (fig. 3, top). In summary: the laser-induced fluorescence spectrum of 3-DF in a supersonic jet is inhomogeneously broadened under conditions when the corresponding spectrum of 3-HF is not. For the deuterated compound, at least three different ground states are responsible for the narrow vibronic bands in the spectrum.
It is interesting at this point to assess the contribution of the residual, undeuterated compound to the spectrum shown in fig. 5B. This may be done by comparing the area of the residual 3-HF band at 28080.3 $\mathrm{cm}^{-1}$ to the areas of the origin bands for the four subsystems. A rough estimates gives a contribution below 15\%, which is in agreement with the MS and NMR data.

\subsection{Fluorescence-dip double-resonance spectroscopy of $3-H F$}

The double-resonance technique described above was also used with undeuterated 3-HF. Here we monitored the fluorescence from excitation in the 00 band at $28080.3 \mathrm{~cm}^{-1}$ (cf. fig. 3, top); the pump laser was set to $28065 \mathrm{~cm}^{-1}$ in the background region. No change was observed when the monitored signal was registered with and without operation of the pump laser.

\section{AM1 calculations of the potential energy surfaces of 3-hydroxyflavone along its torsional coordinates}

In this section we investigate the potential energy surface of 3-HF in its normal tautomeric form, i.e. with an intact 3-hydroxy group, in its $S_{0}$ and $S_{1}$ electronic states. The spectroscopic data yield evidence of a complicated ground state potential energy surface. The causes of these complications are most likely to be found along low-frequency coordinates which involve the motion of the phenyl ring and of the labile hydrogen atom. Among the low-frequency coordinates, the torsion of the phenyl ring (angle $\Phi$, cf. fig. 1) was already shown to be active in the excitation spectra $[13,15]$. It remains to explore the second torsional coordinate of 3-HF, namely the torsion of the hydroxyl group (angle $\Theta$ ) with respect to the chromone unit.

In a previous investigation of the ground state geometry of 3-HF [23] with the semiempirical AM1 method, a phenyl torsional angle $\Phi=29.2^{\circ}$ was obtained. In that study the hydroxyl angle was fixed to $\Theta=0^{\circ}$. A systematic computation in the complete $(\Theta \backslash \Phi)$ plane was carried out to search for other local energy minima. We chose the semiempirical MNDO Hamiltonian with the AM1 parameters [32] 
for our study since it has been very successful in the prediction of molecular geometries and because the task is well beyond the capabilities of present $\mathrm{ab}$ initio methods.

A slightly modified version of the program package MOPAC was used [33]. One option of the program allows the calculation of a grid with equidistant steps in two coordinates and geometry optimization of other selected coordinates. The restricted original grid size of $11 \times 11$ points was enlarged to permit a calculation along a complete rotation of the hydroxyl group and a $90^{\circ}$ rotation of the phenyl group with a stepsize of $10^{\circ}$. This is sufficient for the complete grid since the point $(-\boldsymbol{\theta} \backslash-\Phi)$ constitutes an enantiomer of the point $(\boldsymbol{\theta} \backslash \boldsymbol{\Phi})$, and the phenyl group is symmetric with respect to a rotation by $180^{\circ}$. Normally, the program performs an optimization of specified geometric parameters at each gridpoint taking the starting geometry and density matrix from the previous point. We added an option which, after the geometry optimization, performs a single calculation at this fixed geometry with a more elaborate wavefunction. For all calculations reported here this was a configuration interaction calculation (MOPAC keyword C.I. $=4$ ) which also yields energies for the excited states. The results of these additional calculations were written to a file from which subsequently the grid for the correlated ground state or the excited state could be obtained. Thus a "vertical" excitedstate potential surface was generated. Here "vertical" refers to the fact that all geometrical parameters, except the grid coordinates, have their fixed groundstate values. In order to ensure that the additional calculations have no effect on the grid calculation itself, the geometry optimization at each point was always initialized with the starting geometrical parameters and a new density matrix. Since the calculation of a single gridpoint needed 2-3 min of computer time on an IBM 3090 mainframe, the program was further modified to enable a piecewise calculation of the grid. In the grid-search all bond lengths and bond angles were optimized, but all dihedral angles were fixed so that the phenyl ring and the chromone unit were planar.

The result of this grid calculation is visualized for the correlated ground state in fig. 6 and for the first excited singlet state in fig. 7. The distance of the contour lines is $100 \mathrm{~cm}^{-1}$ in fig. 6 and $200 \mathrm{~cm}^{-1}$ in fig.

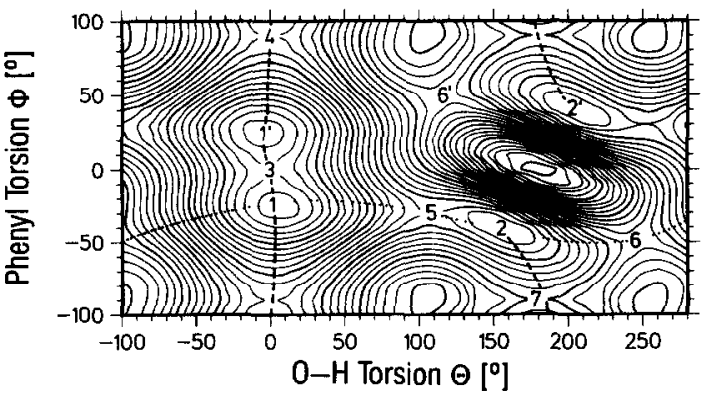

Fig. 6. Contour plot of the ground-state potential energy surface of 3-HF along the two torsional coordinates. All bond lengths and bond angles were optimized on the SCF level and the energy corrected for correlation effects with a limited CI. For details of the calculation see text. Contour lines are separated by $100 \mathrm{~cm}^{-1}$.

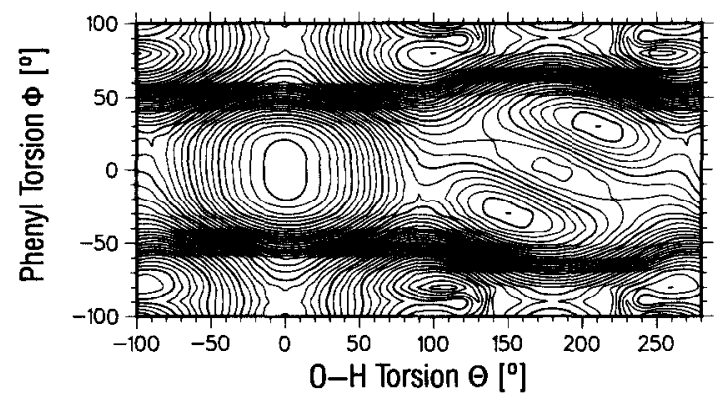

Fig. 7. Contour plot of the "vertical" potential energy surface for the first excited singlet state of 3-HF, obtained from a CI calculation at the fixed ground-state geometries. Contour lines are separated by $200 \mathrm{~cm}^{-1}$.

7. The ground-state energy surface shows two enantiomeric pairs of minima, labelled $1,1^{\prime}, 2$ and $2^{\prime}$ in fig. 6 . The positions of the minima can be localized more precisely if the grid coordinates are also optimized. The result is $E_{1}=-34.878 \mathrm{kcal} / \mathrm{mol}$, $\theta_{1}=-5.72^{\circ}, \Phi_{1}=28.15^{\circ}$, and $E_{2}=-31.706 \mathrm{kcal} /$ mol, $\theta_{2}=160.58^{\circ}, \Phi_{2}=-44.17^{\circ}$. These values change only slightly if the restrictions on the other torsional angles are also lifted (see table 1). The chromone moiety and the phenyl moiety of the molecule remain almost perfectly planar. Performing a configuration interaction calculation $(C . I .=4)$ at these fixed geometries leads to a small stabilization of 2.64 and $2.48 \mathrm{kcal} / \mathrm{mol}$ for the two minima, and an energy difference of $1145 \mathrm{~cm}^{-1}$ is predicted at this level of the theory. An attempt to further improve the optimized geometry on the CI level returned exactly 
the input geometry, which indicated that the geometry had already converged to the optimum on the SCF level.

Inspection of the contour plot reveals that the interconversion of the forms 1 and $1^{\prime}$ should be easiest along a reaction coordinate with a saddle point 3 close to $(\theta=0, \Phi=0)$. This saddle point was localized with the non-linear least-squares gradient minimization technique implemented in MOPAC (keyword NLLSQ). Its energy with respect to point 1 is $\Delta E_{13}=116 \mathrm{~cm}^{-1}$. The path from 1 to $1^{\prime}$ is schematically shown in fig. 6 by a dashed line. It consists mainly of the phenyl-ring torsional coordinate. Hence a deuterium effect on this motion should be very small. A second stationary point which is a maximum along the phenyl ring torsion but a minimum along the coordinate of the motion of the hydroxyl group is marked 4 in the contour plot 6 . Its energy is $1168 \mathrm{~cm}^{-1}$ above minimum 1. This is a rather high barrier, and if the system were one-dimensional one would expect that it be confined to the double-well region with the two equivalent minima 1 and $1^{\prime}$.

The saddle point at lowest energy which permits the system to reach the metastable, local minimum 2 is marked 5 in the contour plot. Its energy is found to be $1292 \mathrm{~cm}^{-1}$. For the reverse process, this corresponds to a barrier of only $147 \mathrm{~cm}^{-1}$ for the conformer at minimum 2 to fall back to the geometry 1 . An alternative path from 2 to 1 leads over the saddle point 6 , which is $1949 \mathrm{~cm}^{-1}$ above 1 and $804 \mathrm{~cm}^{-1}$ above 2 . Finally, the conformers 2 and $2^{\prime}$ could directly exchange via the saddle point 7 at which the phenyl group is perpendicular to the chromone unit.

In the first excited singlet state the coplanar configuration of the phenyl ring and the chromone unit is strongly favored (cf. fig. 7). The potential energy surface rises steeply in the range $50^{\circ}<\Phi<70^{\circ}$. One consequence is that the lowest minimum 1 has the completely planar geometry and no longer appears as a double well. The secondary minimum 2 still exists at $\left(\theta=150^{\circ}, \Phi=-35^{\circ}\right)$, i.e. at a torsional angle of the phenyl group which is smaller by $9^{\circ}$ compared to the electronic ground state. Therefore, vertical excitation from the ground state structure 2 will produce the molecule in the excited state with an excess energy which is larger than the barrier for the decay of 2 to 1.

\section{Discussion}

\subsection{Assessment of the semiempirical calculations}

In assessing the results from our semiempirical calculations, it should be noted that the energy differences between the various stationary points are all small compared to the total electronic and nuclear potential energy of the molecule. Through comparison of the calculated heat of formation for a large number of compounds the average accuracy of the AMl method was found to be $5.88 \mathrm{kcal} / \mathrm{mol}(2057$ $\mathrm{cm}^{-1}$ ) [32]. Even if for a comparison of different molecular systems containing the same number and type of particles a somewhat better agreement can be expected, the energy differences found for $3-\mathrm{HF}$ are too small to allow a quantitative interpretation. What we can say is that there exists the possibility that 3 $\mathrm{HF}$ in its electronic ground state may exist in two forms: a "cis" conformation in which the hydroxyl group is oriented towards the carbonyl oxygen forming a weak intramolecular hydrogen bond, and a "trans" conformation without an intramolecular hydrogen bond. Both forms should exist as pairs of enantiomeric conformers. The cis-pair forms a doubleminimum well ( 1 and $1^{\prime}$ in fig. 6 ) along the phenyl ring torsional coordinate $\Phi$. The barrier between these two wells is probably very low. The trans-pair 2 and $2^{\prime}$ is separated by a higher barrier (saddle point 7 in fig. 6) at a true trans-conformation for the hydroxyl group, and with the phenyl ring perpendicular to the chromone moiety. The lowest energy path for interconversion $2 \leftrightarrow 2$ ' follows a correlated, "geared" motion along both torsional coordinates.

The task before us, then, is to explain the experimental observations, namely (i) the coexistence of vibronic structure and spectral background without a common vibrational ground state, and (ii) the vibronic structure and its complication by $H / D$ substitution. As regards vibronic structure, the following data are available: (a) the pattern of vibronic separations (which applies equally to 3-HF and to 3-DF) indicating excited-state vibrational energy levels, and (b) the different spectral origins of the patterns for 3-DF which reflect differences in ground-state vibrational energy levels for 3-DF but not for 3-HF. Set against these few observations is the large number of parameters for molecular structure and potential en- 
ergy surfaces. In order to make some progress at all, we use the results of our semiempirical calculations in a qualitative way. Thus we adopt the hypothesis that in a supersonic jet, a measurable fraction of the molecular population may be trapped in the (approximately) trans-conformation for the hydroxyl group with respect to the carbonyl group. This hypothesis retains the qualitative results of our semiempirical calculations but disregards the calculated relative energetics and stability of trans- versus cisisomers. In the following we investigate whether this hypothesis is consistent with observations.

\subsection{The spectral background}

A spectral background to laser-induced fluorescence in the supersonic jet was observed both for the deuterated and nondeuterated compound; hence the two observations (i) and (ii) appear to be unrelated. The background may have several causes, like fluorescing impurities, complexation with $\mathrm{H}$-bonding molecules such as water or methanol [13], complexation with the noble gas which is used for supersonic expansion, and spectral congestion of hot bands. From our procedure for sample preparation and the stability of the 3-DF spectra over longer measurement times we feel that sample impurities or water complexes are unlikely to be the causes for the observed spectral background in our case. The background persisted or even increased when neon as carrier gas was replaced by helium which is less likely to form complexes. In addition, molecules of similar size had not shown measurable complexation with neon for the stagnation conditions which were employed in our experiments. As regards hot bands for 3-HF, there should be some residual structure to the red of the 00 transition, at least for moderate stagnation pressure; the spectral density in this region due to hot bands should in principle be of the same order as for the molecule in its vibrational ground state. Both inferences are contrary to observation. We have never obtained a ratio of band amplitude/background better than 10 for the 00 transition in 3-HF, nor has such a claim been made in the literature. The spectral integral over the background signal exceeds the integral signal which can be attributed to the structured spectrum. In summary it appears that the background is an intrinsic and significant property of jet-cooled, bare 3-hydroxyflavone.

Now consider the hypothesis that background and vibronic structure may belong to different isomers of the molecule in the ground state. As one possibility, the pronounced vibronic bands of 3-HF in fig. 3 might belong to a trans-isomer (labelled 2 in fig. 6). This assignment in turn demands that the unstructured background be assigned to the normal cis-isomer with an intramolecular hydrogen bond (labelled 1 in fig. 6 ). In the remainder of this section, we shall concentrate on the normal cis-isomer.

A lower energy for the 00 transition of the cis-isomer is reasonable and consistent with our semiempirical calculations. At an excess vibrational energy of more than $\approx 200 \mathrm{~cm}^{-1}$ the vibronic structure of the cis-isomer should be congested (torsional activity of the phenyl ring in combination with other lowfrequency modes ). In addition, a fast intramolecular proton transfer reaction with time constant on the order of $240 \mathrm{fs}$ [22] implies a homogeneous linewidth around $22 \mathrm{~cm}^{-1}$. Congestion and linewidth together would indeed produce an unstructured background at excess vibrational energies of several hundred $\mathrm{cm}^{-1}$ for cis- and at the first vibronic band of the transisomer. The homogeneous width of the observed vibronic bands, which corresponds to a vibronic lifetime of $\approx 1.5$ ps [15], would then be determined by $-\mathrm{OH}$ torsion rather than by proton transfer. The effect of deuteration on linewidth should be pronounced in this case, as was indeed found in our experiments (cf. fig. 4).

If the conjecture above is correct, then one should find an origin band for excitation of the cis-isomer of 3-HF at energies well below $28080.3 \mathrm{~cm}^{-1}$. However no vibronic structure has so far been discovered in that region. Yet this fact is entirely consistent with our hypothesis as will be shown in the following.

Fig. 8 gives sections of the $S_{0}$ (bottom) and $S_{1}$ (top) potential energy surface for the normal cis-isomer $\left(\theta \approx 0^{\circ}\right)$ along the coordinate for phenyl torsion, $\Phi$. The torsional eigenvalue equation $-B(\Phi) \mathrm{d}^{2} \Psi / \mathrm{d} \Phi^{2}+V(\Phi) \Psi=E \Psi$ was solved numerically [34]. Here, a potential of the form $V(\Phi)=\frac{1}{2} \sum V_{n}[1-\cos (n \Phi)]$ was fitted to the calculated data for the $S_{0}$ and $S_{1}$ states, respectively. The torsional parameter $B$ was calculated from the molecular geometry. The values of all relevant parameters 

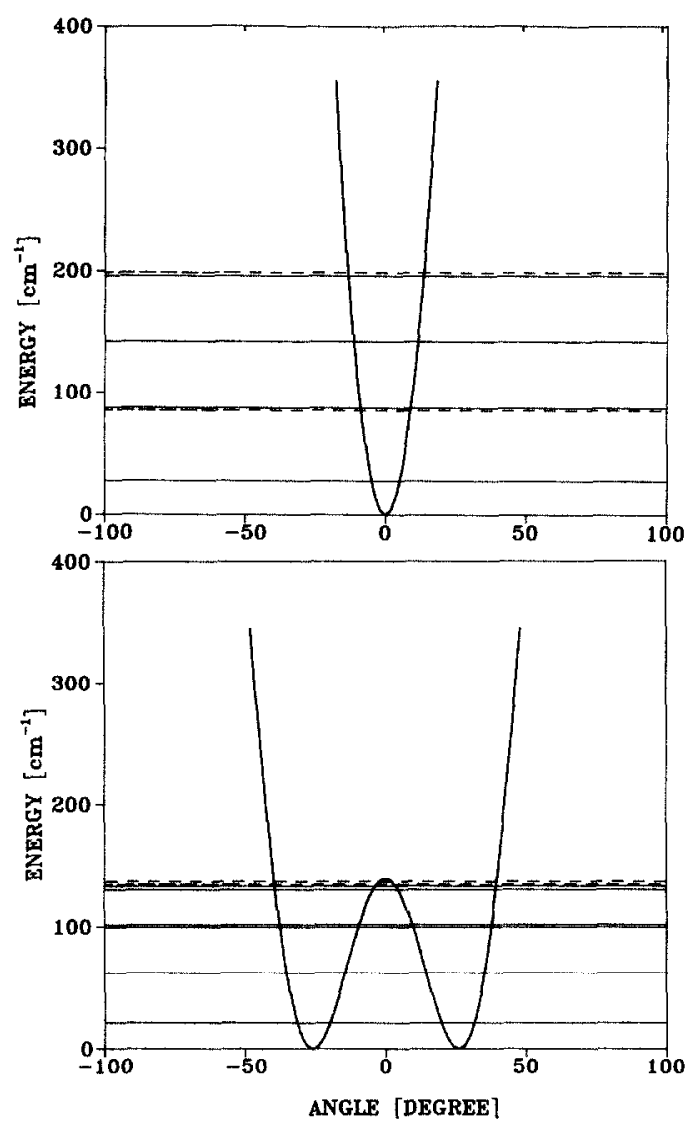

Fig. 8. Phenyl torsion in 3-HF: $S_{0}$ (bottom) and $S_{1}$ (top) electronic states - potential energy curves and the torsional energy levels (see text). Dashed lines indicate energy levels of $a^{\prime \prime}$ symmetry, while solid horzontal lines are used for levels of a' symmetry or for degenerate levels. for this calculation are collected in table 3 . The resulting torsional energy levels are also indicated in fig. 8 .

It can be seen immediately from fig. 8 that optical transitions should appear in a Franck-Condon pattern for phenyl torsion which is widely red-degraded. This qualitative expectation is not significantly affected by reasonable changes in the ground-state double well potential. Therefore a well-defined 00 transition of the cis-isomer may not be observable because of its small Franck-Condon factor in combination with a broad lineshape due to ultrafast intramolecular proton transfer.

\subsection{Vibronic structure}

In our hypothesis, the vibronic structure which is observed in the supersonic jet has been assigned to a trans-isomer (labelled 2 in fig. 6). This vibronic structure need not be identical with that of 3-DF reported in matrix-isolation studies, since the transisomer may not be populated significantly under the conditions for matrix deposition.

Inspection of the potential energy surfaces in the trans-region reveals that there is no qualitative change of the surface in this region upon electronic $S_{0} \rightarrow S_{1}$ excitation; only a shift of the local minimum is predicted. In this case a blue-degraded Franck-Condon pattern is expected. Again this is consistent with the observations (cf. fig. 3, top).

The observed linewidth corresponds to vibronic lifetimes of $1-2 \mathrm{ps}$. The interpretation here is as fol-

Table 2

Optimized angles $\theta$ and $\Phi$ (in degree) for minima and saddle points on the potential energy surface of the electronic ground state of 3 HF. All geometrical parameters were varied in the gradient minimization. $E_{\mathrm{SCF}}$ is the optimized energy for the closed shell wavefunction in $\mathrm{kcal} / \mathrm{mol}, E_{\mathrm{CI}}$ the corresponding energy with a small correlation correction (MOPAC keyword C.I. $=4$ ). $\Delta E$ gives the energy differences with respect to the global minimum, punt 1

\begin{tabular}{lllllll}
\hline Point & $\begin{array}{l}\boldsymbol{\theta} \\
(\mathrm{deg})\end{array}$ & $\begin{array}{l}\boldsymbol{\Phi} \\
(\mathrm{deg})\end{array}$ & $\begin{array}{l}E_{\text {SCF }} \\
(\mathrm{kcal} / \mathrm{mol})\end{array}$ & $\begin{array}{l}E_{\mathrm{CI}} \\
(\mathrm{kcal} / \mathrm{mol})\end{array}$ & $\begin{array}{l}\Delta E \\
\left(\mathrm{~cm}^{-1}\right)\end{array}$ & Method \\
\hline 1 & -5.99 & 28.88 & -34.880 & -37.517 & 0 & SCF \\
2 & 159.52 & -44.65 & -31.759 & -34.241 & 1145 & SCF \\
3 & -0.09 & -0.11 & -34.306 & -37.185 & 116 & NLLSQ \\
4 & 5.39 & 87.62 & -32.792 & -34.174 & 1168 & NLLSQ \\
5 & 108.53 & -32.51 & -31.207 & -33.818 & 1292 & SADDLE \\
6 & 115.75 & 46.92 & -29.587 & -31.939 & 1949 & NLLSQ \\
7 & 179.39 & 89.85 & -30.643 & -31.896 & 1964 & NLLSQ \\
\hline
\end{tabular}


Table 3

Parameters used for the calculation of torsional energy levels (all in $\mathrm{cm}^{-1}$ ) [34]

\begin{tabular}{lllllrl}
\hline & \multicolumn{2}{l}{ Potential parameters } & & & \\
\cline { 2 - 6 } & $V_{1}$ & $V_{2}$ & $V_{3}$ & $V_{4}$ & $V_{5}$ & $V_{6}$ \\
\hline $\mathrm{S}_{0}$ & -2.09 & 1097.32 & -1.40 & -361.93 & -1.69 & -133.96 \\
$\mathrm{~S}_{1}$ & -26.63 & 4834.52 & -16.43 & -345.42 & -19.13 & \\
torsional constant & $B=0.223$ & & & & & \\
\hline
\end{tabular}

lows: a fast (ps) rotation of the hydroxyl group around the HO-C bond forms the cis-isomer in its $\mathrm{S}_{1}$ state; the electronically excited cis-isomer reacts by ultrafast ESIPT to the excited tautomeric molecule, which finally relaxes by tautomeric fluorescence. The hydroxyl rotation may be fast due to tunneling. Indeed $H / D$ substitution lead to a significant lengthening of vibronic lifetimes. In any case (that is, $3-\mathrm{HF}$ or 3-DF) vibronic lifetimes on the order of $10 \mathrm{ps}$ or less should correspond to a quantum yield for normal fluorescence which is smaller by a factor $5 \times 10^{-3}$ compared to the quantum yield for tautomeric fluorescence. For such relative quantum yields our experimental conditions are not adequate to record the corresponding normal fluorescence from the primary excited vibronic states. Thus the lack of observed primary fluorescence is still consistent with our hypothesis.

\subsection{Spectral complexity upon $H / D$ exchange}

The inhomogeneous complication of the structured spectrum which is caused by H/D substitution (cf. fig. 3 ) is the most solid of our observations and, at the same time, the most difficult to explain. Three interpretations come to mind. First, H/D substitution may take place at three different positions. However this possibility is refuted by the NMR results which clearly indicate that only the hydroxylic hydrogen is replaced. Second, the transitions in 3-HF may be degenerate (with more than two components) and this degeneracy is removed in 3-DF. Third, deuteration may lead to a regrouping and concentration of vibrational levels at low energies. As a result, hot bands should now appear which were absent in the undeuterated compound.

Within our hypothesis, the spectral complexity upon H/D substitution should be carried by the transisomer. This isomer - if it exists at all - should perform a geared torsional motion of the phenyl ring and the hydroxyl group against the chromone moiety. This vibrational problem is capable of yielding an energy level structure which is sufficiently complex and which changes upon deuteration; further work on this is in progress in our laboratory. At this point we conclude that the hypothetical assignments is still consistent with our observations.

\section{Conclusion}

The fluorescence excitation spectroscopy of jetcooled 3-hydroxyflavone and its deuterated derivative resulted in the following observations:

Deuteration leads to a narrowing of vibronic bands and to a significant complication of the vibronic spectrum. The latter consists of an inhomogeneous superposition of partial spectra with identical vibrational structure but with different origins. Both the deuterated and undeuterated compound exhibit a broad background to fluorescence excitation in the supersonic jet. The optical transitions corresponding to the background and to the vibronic bands do not share a common ground state.

As a working hypothesis, the possibility of two isomers in the electric ground state of 3-HF in a supersonic jet was explored. Further work is definetely required in order to explain our results.

\section{Acknowledgement}

We are grateful to Dr. K.H. Grellmann for many intensive discussions. This work was supported by the 
Deutsche Forschungsgemeinshaft in the Sonderforschungsbereich 357 (Molekulare Mechanismen Unimolekularer Reaktionen). We also thank the "Fonds der Chemischen Industrie" for support. Most of the MOPAC calculations and the contour plots were performed with resources provided by the "Gesellschaft für wissenschaftliche Datenverarbeitung Göttingen".

\section{References}

[1] T. Swain, in: Chemistry and biochemistry of plant pigments, ed. T.W. Goodwin (Academic Press, New York, 1976) ch. 20.

[2] A. Weller, Z. Electrochem. 60 (1956) 1144.

[3] P.F. Barbara, P.K. Walsh and L.E. Brus, J. Phys. Chem. 93 (1989) 29

[4] P.K. Sengupta and M. Kasha, Chem. Phys. Letters 68 (1979) 382.

[5] M. Itoh and H. Kurokawa, Chem. Phys. Letters 91 (1982) 487.

[6] O.A. Salman and H.G. Drickamer, J. Chem. Phys. 75 (1981) 572

[7] D. McMorrow and M. Kasha, J. Phys. Chem. 88 (1984) 2235.

[8] D. McMorrow and M. Kasha, Proc. Natl. Acad. Sci. USA 81 (1984) 3375 .

[9] B. Dick and N.P. Ernsting, J. Phys. Chem. 91 (1987) 4261.

[10] G.A. Brucker and D.F. Kelley, J. Phys. Chem. 91 (1987) 2856.

[11] M.C. Etter, Z. Urbanczyk-Lipkowska, S. Baer and P.F. Barbara, J. Mol. Struct. 144 (1986) 155.

[12] G.A. Brucker and D.F. Kelley, J. Phys. Chem. 93 (1989) 5179.

[13] A. Ito, Y. Fujiwara and M. Itoh, J. Chem. Phys. 96 (1992) 7474.

[14] W.E. Brewer, S.L. Studer, P.T. Chou and E. Orton, Chem. Phys. Letters 158 (1989) 345.
[15] N.P. Ernsting and B. Dick, Chem. Phys. 136 (1989) 181.

[16] A. Peluso, C. Adamo and G. Del Re, J. Math. Chem. 10 (1992) 249.

[17] A.J.G. Strandjord and P.F. Barbara, J. Phys. Chem. 89 (1985) 2355.

[18] T.C. Swinney and D.F. Kelley, J. Phys. Chem. 95 (1991) 10369.

[19] G.A. Brucker, T.C. Swinney and D.F. Kelley, J. Phys. Chem. 95 (1991) 3190.

[20] G.A. Brucker and D.F. Kelley, J. Phys. Chem. 92 (1988) 3805.

[21] C. Rulliere and A. Declemy, Chem. Phys. Letters 134 (1987) 64.

[22] B.J. Schwartz, L.A. Peteanu and C.B. Harris, J. Phys. Chem. 96 (1992) 359

[23] B. Dick, J. Phys. Chem. 94 (1990) 5752.

[24] G.J. Woolfe and P.J. Thistlethwaite, J. Am. Chem. Soc. 103 (1981) 6916

[25] M. Itoh, K. Tokumura, Y. Tanimoto, Y. Okada, H. Takeuchi, K. Obi and I. Tanaka, J. Am. Chem. Soc. 104 (1982) 4146.

[26] O.A. Salman and H.G. Drickamer, J. Chem. Phys. 77 (1982) 3329.

[27] M. Itoh and Y. Fujiwara, J. Phys. Chem. 87 (1983) 4558.

[28] M. Itoh, J. Phys. Chem. 90 (1986) 5672.

[29] W.E. Brewer, S.L. Studer, M. Standiford and P.T. Chou, J. Phys. Chem. 93 (1989) 6088

[30] J. Sepiol and R. Kolos, Chem. Phys. Letters 167 (1990) 445.

[31] T.P. Dzugan, J. Schmidt and T.J. Aartsma, Chem. Phys. Letters 127 (1986) 336.

[32] M.J.S. Dewar, E.G. Zoebisch, E.F. Healy and J.J.P. Stewart, J. Am. Chem. Soc. 107 (1685) 3902.

[33] QCPE Program No. 445.

[34] J.D. Lewis, T.B. Malloy, T.H. Chao and J. Laane, J. Mol. Struct. 12 (1972) 427. 